\title{
Fracture of a cemented modern titanium alloy tibia baseplate: a case report
}

\begin{abstract}
This case highlights a fracture of a modern tibia basetray. The patient is a 52-yearold female who underwent a total knee arthroplasty that had catastrophic posterior medial polyethylene wear 5-years following the original surgery. She underwent a polyethylene exchange to an anterior constrained style polyethylene and a year and a half later presented with severe medial pain and a locked knee. Radiographs initially demonstrated significant anterior translation of the tibia on the femur and she was indicated for revision surgery. Radiographs the day prior to revision demonstrate a fractured posterior medial corner of the base tray and a disengaged polyethylene in the suprapatellar pouch. Revision TKA was undertaken and given the inability to control AP translation with a none or semi constrained knee design, reconstruction with a rotating hinge knee was performed.
\end{abstract}

Keywords: tibia tray fracture, AP instability, revision knee, rotating hinge knee
Volume 10 Issue 4 - 2018

\author{
Daniel R Mesko DO,' Alex J Sadauskas BS, ${ }^{2}$ \\ Scott M Sporer MD' \\ 'Department of Orthopaedic Surgery, RUSH University Medical \\ Center, Illinois \\ ${ }^{2}$ Saint Louis University School of Medicine, Missouri
}

Correspondence: Daniel R Mesko DO, Department of Orthopaedic Surgery, RUSH University Medical Center,Address: I6I I W Harrison St, Chicago IL 606 I 2, USA, Tel 877632 6637, Emaildan.mesko@gmail.com

Received: August 05, 2018| Published: August 13, 2018

\section{Introduction}

Total knee arthroplasty (TKA) remains an extremely successful and popular surgery with over 3-million cases projected to be performed annually by $2030 .{ }^{1}$ Although revision rate is low overall, there remains numerous factors that can predispose to failure related to the patient, surgeon, implant, or any combination of these. ${ }^{2}$ Revision related to component failure itself is typically multifactorial in regards to implant material and design along with surgical technique and alignment. Failure related to fracture of a tibia tray is exceedingly rare. As of 2005, 74 cases had been reported in the literature. ${ }^{3}$ This number has increased slightly but remains a rare finding with few cases being described since that time frame, and even less so with a contemporary component. ${ }^{4-8}$ This case report represents, to our knowledge, the first case report of a Zimmer ${ }^{\circledR}$ NexGen ${ }^{\circledR}$ tibia tray fracture, 77 months from the index operation.

\section{Case history}

A 52-year-old woman underwent a left total knee arthroplasty 7-years prior with a cemented CR Zimmer ${ }^{\circledR}$ NexGen ${ }^{\circledR}$ total knee system (Zimmer-Biomet, Warsaw, IN) secondary to rheumatoid arthritis. She had a history of rheumatoid arthritis, lymphoma, COPD, deep vein thrombosis as well as obesity $\left(5^{\prime} 5^{\prime}, 226 \mathrm{Lbs}\right.$, BMI 37). She presented for follow up 5-years after her original surgery with symptoms of pain, difficulty ambulating, and an audible clunk. She demonstrated abnormal AP mechanics with significant anterior tibia translation. She was noted to have near complete wear through the posterior medial corner of her polyethylene (Figure 1) and was subsequently revised to $14 \mathrm{~mm} \mathrm{AC}$ polyethylene with resolution of her abnormal mechanics (Figure 2).

She did well for a year and a half following her revision surgery then presented with knee pain located medially on the joint line, over the pes bursae as well as along her distal IT-band. It was also noted in flexion that her excess soft tissue from her posterior thigh impinged on her posterior leg. Radiographs were obtained (Figure 3), and revision surgery to a hinge type knee was recommended to control AP instability, particularly given the posterior impingement of her soft tissues in the popliteal region secondary to her body mass distribution, which on exam appeared to be causing anterior tibia translation and likely contributing to the catastrophic posterior medial tibia failure.
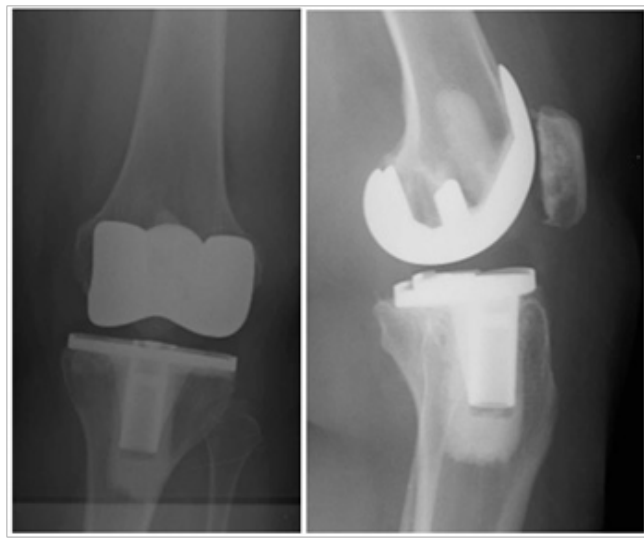

Figure I AP/Lateral left knee 5years from TKA with medial joint space narrowing and anterior translation of the tibia.

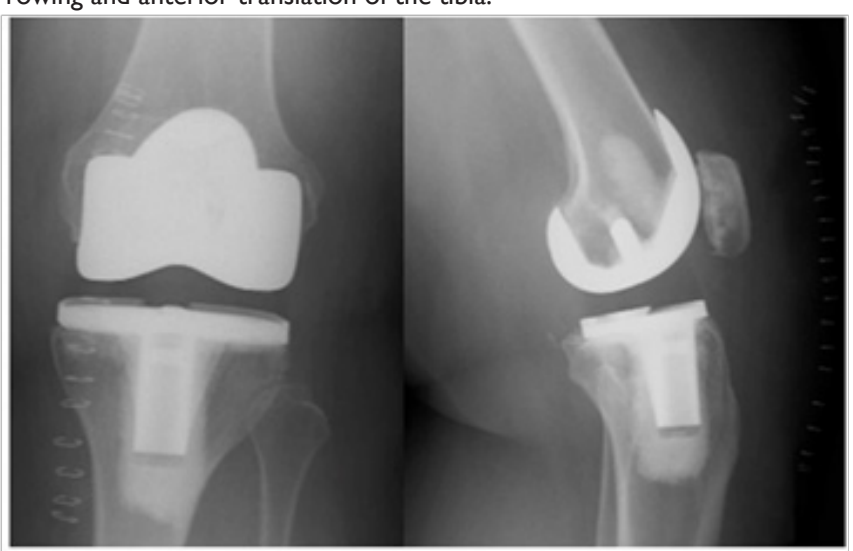

Figure $2 \mathrm{AP} /$ Lateral left knee, status post revision TKA to a $14 \mathrm{~mm}$ AC constrained polyethylene with resolution of joint space asymmetry and appropriate alignment on the lateral view. 


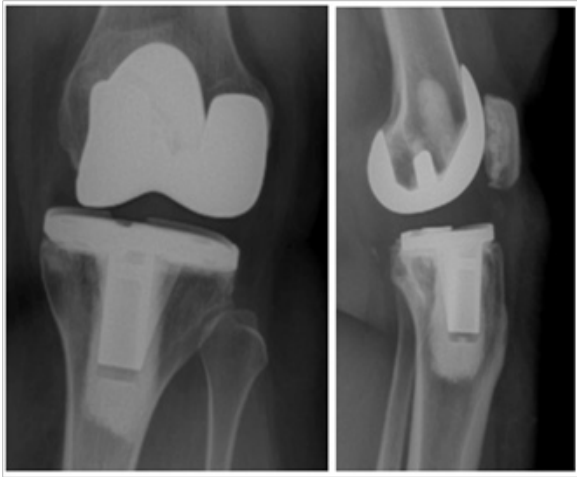

Figure 3 AP/Lateral left knee, I6months following revision surgery, again demonstrating anterior tibia translation and joint line asymmetry concerning for mechanical failure of the polyethylene.

The patient waited, given her symptoms at this time frame were only mild; however, 4 months later she presented for follow up, as she was having significant instability (Figure 4). She was then scheduled for revision TKA.

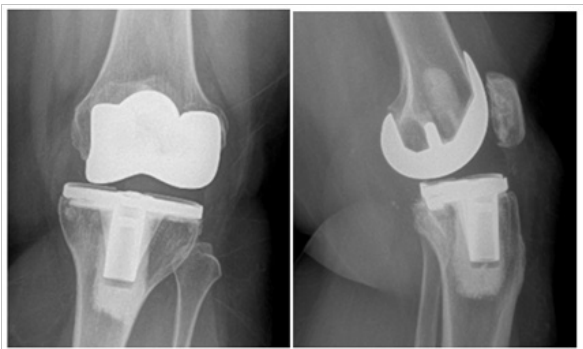

Figure $4 \mathrm{AP} /$ Lateral left knee 20months from revision surgery demonstrating increased anterior tibia subluxation and medial joint space narrowing. There is also evidence of posterior medial tibia tray fracture.

Prior to the patient's chosen surgical date, she twisted her leg, felt a shift in her knee, and had severe pain along with the sensation her knee being locked (Figure 5).

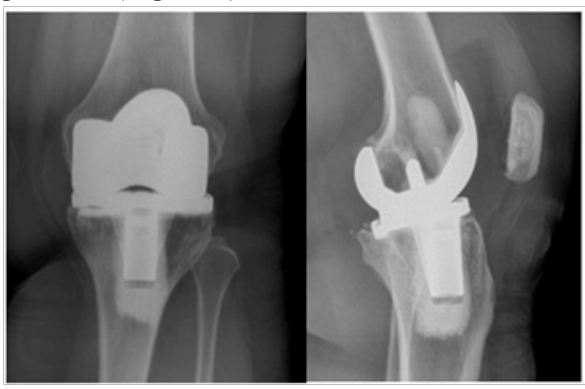

Figure 5 AP/Lateral Left Knee showing metal on metal articulation of the femur on the tibia, disengaged polyethylene in the suprapatellar pouch as well as fracture of the posterior medial corner of the tibia tray.

She was taken to the operating room the following day. Her polyethylene was found in the suprapatellar pouch with damage particularly to the posterior medial region (Figure 6). The tibia tray was found to be well fixed but was found to be fractured in the posterior medial corner (Figure 7). Both component revision was undertaken using Zimmer ${ }^{\circledR}$ NexGen ${ }^{\circledR}$ RH knee with cemented stems as well as a trabecular metal femoral cone in the metaphysis (Figure 8).

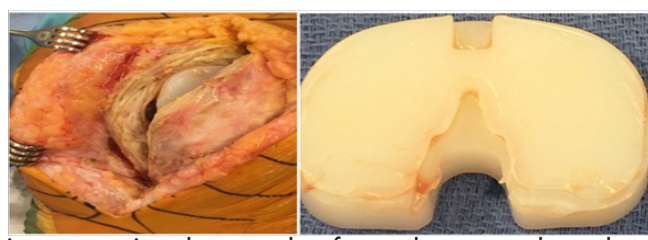

Figure 6 intraoperative photographs after arthrotomy where the polyethylene was encountered within the suprapatellar pouch. Severe posterior damage to the polyethylene was noted.

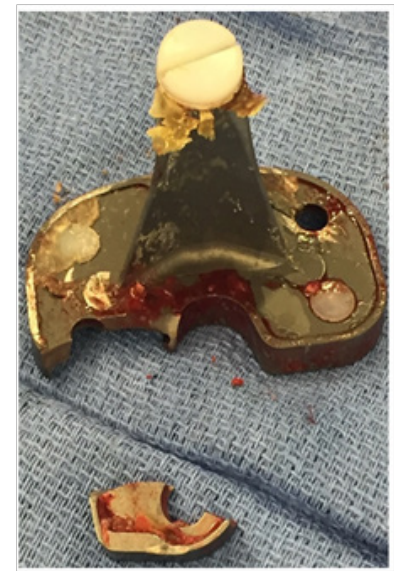

Figure 7 Intraoperative photograph showing fracture of the posterior medial corner of the tibial tray, medially, through a hole which allows for optional augment attachment and into the PCL recess of the tray.

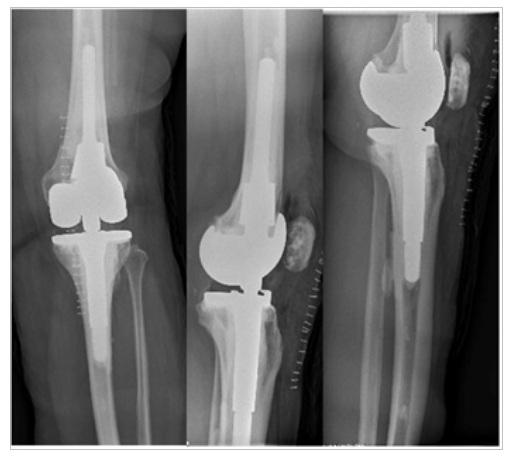

Figure $8 \mathrm{AP} /$ Lateral femur/Lateral tibia of the left knee following revision to a rotating hinge knee with cemented stems and femoral metaphysical cone.

\section{Discussion}

Fractures of the tibia base tray have been described as a complication in TKA dating as far back as $1984 .{ }^{9}$ Metallurgy and stress risers in the design of trays, have been described as contributing factors to the fracture of the tray in multiple case reports..$^{9-14}$ Despite the improvement in technology, there still have been case reports where modern cemented baseplate fractures are encountered. Callaghan et al published on a case of a PFC Sigma (DePuy, Warsaw, IN) baseplate fracture 17 -months post operatively. ${ }^{4}$ The mechanism was postulated to be fatigue fracture due to loss of proximal tibial bone support. ${ }^{4}$ Ho et al., ${ }^{15}$ described a case of a Duracon (Howmedica) basetray fracture at 3years in the setting of significant femoral component malposition. ${ }^{15}$ Mineta et al discussed a case of an Miller Galante II (Zimmer) tibial baseplate fracture 16-years after initial knee arthroplasty, ${ }^{5}$ the complication ultimately attributed to varus 
malalignment of the tibial basetray. Another report on Miller Galante II (Zimmer) tibial baseplate fracture was attributed to under sizing the tray, malrotation, and deficient proximal tibia bone stock. ${ }^{16}$

Obesity has also been described as a separate risk factor for aseptic loosening, varus collapse, or even tray fracture. ${ }^{2,9,14,17-20}$ Abdel et al., ${ }^{17}$ reported the chance of aseptic loosening in TKA patients $\geq 35 \mathrm{~kg} / \mathrm{m}^{2}$ to be nearly double that of those $<35 \mathrm{~kg} / \mathrm{m}^{2}$, independent of patient's age or coronal alignment of the components. ${ }^{17}$ Fehring et al determined that obese individuals undergoing TKA have an increased risk of varus collapse of the tibial component, especially those with smaller sized proximal tibias. ${ }^{18}$ The highest level of bone strain in this cohort was found at the medial aspect of the tibia. Berend et al further associated obesity and varus tibial component alignment with increased medial tibia bone collapse. ${ }^{2}$ This association correlates with our study involving an isolated poster medial tibia base tray fracture.

To our knowledge, there have been no published cases on a Zimmer ${ }^{\circledR}$ NexGen ${ }^{\circledR}$ CR tibia tray fracture. A 2015 study reported on 761 consecutive Zimmer ${ }^{\circledR}$ NexGen ${ }^{\circledR}$ CR TKAs showing a 97.8\% survival rate with revision as an endpoint at 10 -years. ${ }^{21}$ Of the 17 revisions identified in this series, 5 were infection related, 4 aseptic loosening, 3 instability, 2 severe pain, 2 periprosthetic femur fracture and 1 periprosthetic proximal tibia fracture.

\section{Summary}

In this case, the holes in this modern tray, designed for augments, were a weak point in the design and after asymmetric loading, led to fracture of the tray itself. The BMI of 37, which had significant distribution in the posterior thigh and calf regions, led to not only increased load on the tibial tray itself, but also was a potential driving force for the tibia to translate anterior, thus loading the posterior tibia. These factors combined, likely contributed to this current failure. Catastrophic tibia failure secondary to AP instability was managed here utilizing a rotating hinged knee arthroplasty.

\section{Acknowledgements}

None.

\section{Conflict of interest}

The author declares that there is no conflict of interest.

\section{References}

1. Kurtz S, Ong K, Lau E, et al. Projections of primary and revision hip and knee arthroplasty in the United States from 2005 to 2030. J Bone Joint Surg Am. 2007;89(4):780-785.

2. Berend ME, Ritter MA, Meding JB, et al. Tibial component failure mechanisms in total knee arthroplasty. Clin Orthop. 2004;428:26-34.

3. Chatterji U, Ashworth MJ, Smith AL, et al. Retrieval study of Tibial Baseplate fracture after total knee arthroplasty. $J$ Arthroplasty. 2005;20(1):101-107.
4. Callaghan JJ, DeMik DE, Bedard NA, et al. Tibial tray fracture in a modern prosthesis with retrieval analysis. Arthroplasty Today. 2018;10;4(2):143-147.

5. Mineta K, Okada M, Matsumoto S, et al. Fracture of the tibial baseplate 16years after miller-galante II total knee arthroplasty. Case Rep Orthop. 2017;2017:4080816

6. Kang JY, Lee YS. Same-level fracture of the tibial metal tray and polyethylene insert after total knee arthroplasty. Orthopedics. 2016;39(4):e787-789.

7. O'Neill BJ, Cleary M, McElwain JP. Fatigue fracture of tibial arthroplasty implant masked by contralateral knee arthritis. Int J Surg Case Rep. 2013;4(5):496-499.

8. da Palma IM, Albuquerque RPE, Barretto JM. Fracture of the Tibial component in total knee arthroplasty: report on two cases. Rev Bras Ortop. 2011;46(3):325-328.

9. Scott RD, Ewald FC, Walker PS. Fracture of the metallic tibial tray following total knee replacement. Report of two cases. $J$ Bone Joint Surg Am. 1984;66(5):780-782

10. Gradisar IA, Hoffmann ML, Askew MJ. Fracture of a fenestrated metal backing of a tibial knee component: A case report. J Arthroplasty. 1989;4(1):27-30.

11. Mendes DG, Brandon D, Galor L, et al. Breakage of the metal tray in total knee replacement. Orthopedics. 1984;7(5):860-862.

12. Dannenmaier WC, Haynes DW, Nelson CL. Granulomatous reaction and cystic bony destruction associated with high wear rate in a total knee prosthesis. Clin Orthop. 1985;198:224-230.

13. Ranawat CS, Johanson NA, Rimnac CM, et al. Retrieval analysis of porous-coated components for total knee arthroplasty. A report of two cases. Clin Orthop. 1986;209:244-248.

14. Morrey BF, Chao EY. Fracture of the porous-coated metal tray of a biologically fixed knee prosthesis. Report of a case. Clin Orthop. 1988;228:182-189.

15. Ho T-F, Tsai R-Y, Lee P-Y, et al. Early tibial tray failure of a duracon knee with retrieval analysis1. J Arthroplasty. 2004;19(6):797-802.

16. Altintas F, Sener N, Ugutmen E. Fracture of the tibial tray after total knee arthroplasty. J Arthroplasty. 1999;14(1):112-114.

17. Abdel MP, Bonadurer GF, Jennings MT, et al. Increased aseptic tibial failures in patients with a BMI $\geq 35$ and Well-Aligned Total Knee Arthroplasties. J Arthroplasty. 2015;30(12):2181-2184.

18. Fehring TK, Fehring KA, Anderson LA, et al. Catastrophic varus collapse of the tibia in obese total knee arthroplasty. $J$ Arthroplasty. 2017;32(5):1625-1629.

19. Swarts E, Miller SJ, Keogh CV, et al. Fractured whiteside ortholoc II knee components. J Arthroplasty. 2001;16(7):927-34.

20. Cankaya D, Yilmaz S, Deveci A, et al. Tibial baseplate fracture associated with polyethylene wear and osteolysis after total knee arthroplasty. Int J Surg Case Rep. 2014;5(2):49-51.

21. Hack J, Mai S, Siebert W. 10-Year Follow-Up of the NexGen CR Total Knee Prosthesis. Z Orthopadie Unfallchirurgie. 2015;153(5):508-515. 